\title{
HIGHER MASS SCALES AND MASS HIERARCHIES
}

\author{
Thomas APPELQUIST, T. TAKEUCHI \\ Center for Theoretical Physics, Department of Physics, Yale University, New Haven, CT 06511, USA \\ Martin EINHORN \\ Department of Physics, University of Michigan, Ann Arbor, MI 48109, USA
}

and

L.C.R. WIJEWARDHANA

Department of Physics, University of Cincinnati, Cincinnati, OH 45221, USA

Received 15 February 1989

\begin{abstract}
The impact of new interactions at very high energies on the spontaneous breaking of chiral symmetry in gauge field theories is studied. Described at low energies by higher dimension operators, these interactions can enhance the chiral condensate and even the Goldstone boson decay constant relative to the confinement scale. This can lead to important consequences in technicolor theories, including realistically large quark and lepton masses and the possibility of new physics at energies below the weak scale. In addition, small differences in strength of the four-fermion interactions, due say to isospin or flavor breaking, can be greatly magnified in the resulting quark and lepton mass spectrum.
\end{abstract}

In this letter, we summarize a study of the effect of new, high energy interactions on the dynamical breaking of chiral symmetry in asymptotically free gauge field theories, in particular technicolor theories. We will show that these interactions, described at low energies by higher dimension operators, can have an important effect on the mass scales that emerge from the breaking.

In asymptotically free theories, the running coupling $\alpha(p)$ is strong at momenta on the order of the confinement scales $A_{\mathrm{c}}$. It then decreases like $1 / \ln (p)$ until it reaches some smaller value $\alpha(\Lambda)$, where $\Lambda$ is the cutoff beyond which the theory can no longer be used in isolation. The value of $\alpha(A)$ is determined by the unknown high energy physics that lurks beyond $A$. Dynamical chiral symmetry breaking, if it is driven purely by the gauge interaction, can only take place when $\alpha(p)$ exceeds some critical value $\alpha_{\mathfrak{c}}[1]$, expected to be reasonably strong $\#$. Thus if $\alpha(\Lambda) \ll \alpha_{c}$, the breaking must take place at scales well below the cutoff and perhaps on the order of the confinement scale $A_{\mathrm{c}}$. In QCD this is precisely what happens, and technicolor theories are expected to behave in a similar way. The Goldstone boson decay constants for each case are $f_{\pi}=93 \mathrm{MeV}$ and $F=\mathrm{O}(250$ $\mathrm{GeV}$ ).

In either case, the new physics beyond $\Lambda$ can be described at low energies in terms of effective higher dimensions operators. The dominant contribution at low energies should arise from dimension six, fourfermion operators. In technicolor theories, these operators (ETC interactions) [3] ${ }^{\# 2}$, treated as perturbations to the dominant technicolor gauge interactions, give rise to quark and lepton masses, pseudoGoldstone boson masses, and typically to flavor-

\footnotetext{
\#1 There is some evidence, however, that the ladder expansion may be reasonably convergent for $\alpha=\mathrm{O}\left(\alpha_{\mathrm{c}}\right)$; see ref. [2].

\#2 For a recent review of technicolor see ref. [4].
} 
changing neutral currents among the quarks and leptons.

Without some GIM-type mechanisms, they must be suppressed by taking $A$ to be at least several hundred $\mathrm{TeV}$, leading to a potential problem for technicolor theories: Quark and lepton masses are given by $m(\mathrm{q}, \ell) \sim\left(g^{2} / \Lambda^{2}\right)\langle\bar{\psi} \psi\rangle$, where $g^{2}$ is a dimensionless coupling strength and $\langle\bar{\psi} \psi\rangle$ is the technicolor condensate, naively expected to be of order $A_{\mathrm{c}}^{3}$. These masses then turn out to be no more than a fraction of an $\mathrm{MeV}$. This difficulty can be mitigated in technicolor theories with a walking coupling [5] ${ }^{\# 3}$, in which $\langle\bar{\psi} \psi\rangle$ can be enhanced by up to nearly two orders of magnitude [8] ${ }^{\sharp 4}$. Even this effect, however, does not seem adequate to produce a realistic quark and lepton mass spectrum.

The essential point of this letter is that the higher energy, four-fermion interactions can also have an important direct effect on chiral symmetry breaking in gauge field theories, both walking and normally running, leading for example to realistically large quark and lepton masses. If these interactions are strong enough, they cannot be treated as perturbations to the gauge interactions. Instead, they must be included in the gap equation that governs the breaking and they can dramatically affect the mass scales that emerge. That four-fermion interactions can drive dynamical breaking has of course been known since the classic paper of Nambu and Jona-Lasinio [10]. If they begin to dominate the dynamics in the present framework, however, the low energy expansion could be breaking down and even higher dimension operators could then play a role. As we present our results, we will discuss this point. For economy, the discussion from here on will be in the context of technicolor.

To begin, we neglect flavor symmetry violation. The technifermions together with their technicolor and

\footnotetext{
\#3 The idea of raising the fermion condensate was first discussed by Holdom [6]. To achieve this he imagined that the theory might have an ultraviolet stable fixed point. In an effort to understand monojet events once suggested by the UAl group at CERN, he also examined slowly running theories [7] to try to produce a hierarchy between the confinement and chiral breaking scales. He noted that such theories can also enhance fermion masses.

\#4 Higher condensate enhancements can be obtained if one assumes the coupling to remain constant all the way to the ETC cutoff.
}

four-fermion interactions will then respect a global $\mathrm{SU}\left(N_{\mathrm{f}}\right) \times \mathrm{SU}\left(N_{\mathrm{f}}\right)$ symmetry. The breaking will lead to a residual $\mathrm{SU}\left(N_{\mathrm{f}}\right)_{\mathrm{L}+\mathrm{R}}$ symmetry with a dynamical technicolor-singlet fermion mass $\Sigma(p)$ determined by an appropriate gap equation. With the gauge sector treated in dressed ladder approximation ${ }^{\sharp 5}$, the gap equation will take the form

$$
\begin{gathered}
\Sigma(p)=\frac{1}{4} \int_{0}^{\Lambda^{2}} \frac{k^{2} \mathrm{~d} k^{2}}{M^{2}(k, p)} \frac{\alpha(M)}{\alpha_{c}} \frac{\Sigma(k)}{k^{2}+\Sigma^{2}(k)} \\
+2 \frac{g^{2}}{\Lambda^{2}}\left(\frac{1}{4 \pi^{2}} \int_{0}^{A^{2}} k^{2} \mathrm{~d} k^{2} \frac{\Sigma(k)}{k^{2}+\Sigma^{2}(k)}\right) .
\end{gathered}
$$

The first term is the technicolor contribution in Landau gauge, $M(k, p)$ is the maximum of $k$ and $p$, and $\alpha_{c}=\pi / 3 C_{2}$ is the critical gauge coupling required for spontaneous breaking in the absence of other interactions. $C_{2}$ is the quadratic Casimir of the technifermion representation. The second term comes from the chirally invariant four-technifermion interaction. Its coupling strength is defined to be $g^{2} / \Lambda^{2}$, and it is assumed that the physics giving rise to this interaction damps rapidly beyond $\Lambda$. It is also assumed that the four-fermion interaction is attractive in the technicolor-singlet channel. Factors arising from the closed loop sum over $N_{\mathrm{f}}$ and technicolor have been absorbed into $g^{2}$. The quantity in square brackets, when multiplied by the dimensionality of the technifermion representation, is the technifermion condensate.

A qualitative picture of how $\Sigma(p)$, and also $F$ and the condensate $\langle\bar{\psi} \psi\rangle$, depend on the four-fermion strength $\lambda \equiv g^{2} / 2 \pi^{2}$ is not difficult to come by. For very small $\lambda$, the physics will be dominated by the technicolor interaction. $\Sigma(p)$ will be of order $A_{c}$ for $p \sim \Lambda_{\mathrm{c}}$. For $p \gg \Lambda_{\mathrm{c}}$, it will fall with increasing $p$ at a rate roughly between $1 / p$ and $1 / p^{2}$ depending on the rate of running of the coupling. The integral expression giving $F$ in terms of $\Sigma(p)[11]^{\# 6}$ converges rapidly for any rate of fall of $\Sigma(p)$ in the above range,

\#5 In this improved version of the ladder approximation vertices and propagators are dressed by taking all the relevant one loop corrections into account.

\#6 The integral is convergent if $\Sigma(p)$ falls with $p$ as an power. If $\Sigma(p)$ falls logarithmically or does not fall at all as is the NambuJona-Lasinio case, $F \sim \log (A)$. Thus as $\lambda \rightarrow 1, F$ will increase relative to $\Sigma(0)$. 
and leads to a value of $F$ on the order of $A_{c}$. The condensate depends sensitively on the running and can vary from being roughly of order $A_{\mathrm{c}}^{3}$ (ordinary running) to up to two orders of magnitude larger (very slow running).

Suppose next that $\lambda$ is very large, corresponding to the existence of new strong interactions among the technifermions at energies of order $A$. If the gauge interaction is neglected, chiral symmetry breaking will take place only for $\lambda \equiv g^{2} / 2 \pi^{2}>1$. If $\lambda$ is well above 1 , the gauge interaction can be treated as a perturbation and $\Sigma(p)$ will turn out to be of order $\Lambda\left(\gg \Lambda_{c}\right)$ and essentially independent of $p$. In this limit, $F=\mathrm{O}(A)$ and $\langle\bar{\psi} \psi\rangle=\mathrm{O}\left(\Lambda^{3}\right)$. This of course is a completely unphysical limit from the point of view of technicolor. For the reasons described above, a hierarchy at least of order $10^{3}$ must be maintained between $F$ and $A$. It is also a limit in which the low energy expansion breaks down since the physics is taking place dominantly at the cutoff, and the above estimates are not reliable in detail.

It is the range of intermediate values of $\lambda$ that is of interest for technicolor theories. We have studied the evolution of $F$ and $\langle\bar{\psi} \psi\rangle$ throughout this range, both analytically and numerically ${ }^{\sharp 7}$. For most of the range, $\Sigma(0) \ll \Lambda$, and an analytic treatment is possible because the integral equation can be linearized for $p, k \gg \Sigma(0)$. The analytic treatment can then be supplemented numerically for all $\lambda$. The numerical results for a typical case, an SU(4) gauge theory with the number of technifermions varied to adjust the rate of running of the gauge coupling $\#$, are displayed in table 1 . It is only the orders of magnitude of these results and their sensitivity to $\lambda$ that are important. The main features of both the analytic and numerical results are the following:

(1) For essentially any rate of running, the fourfermion term makes a relatively small contribution to $\Sigma(0)$ for most of the range of $\lambda$ between 0 and 1 .

\footnotetext{
\#7 A corresponding analysis for fixed gauge coupling $\alpha$ has been described in ref. [12]. There, a critical curve in the $\alpha, \lambda$ plane, that defines the onset of chiral symmetry breaking, is depicted. An intuitive understanding of chiral symmetry breaking when the coupling $\alpha$ runs can be obtained by referring to this curve. Details will be discussed elsewhere.

$\nexists 8$ The lowest order expression for the $\beta$ function is used here simply to illustrate the effect of different rates of running. Higher order effects are considered in refs. [5-7].
}

For normal running [ $(b-\delta b) \alpha_{c} \sim 1$, where $b$ and $\delta b$ are the gauge and fermion contributions to the beta function $\beta(\alpha)=-(b-\delta b) \alpha^{2}$, it becomes comparable to the technicolor contribution only for $\lambda \simeq 0.93$. For somewhat slower running [ $(b-\delta b) \alpha_{c} \sim 0.4$ ], as might be expected in many technicolor theories, it becomes comparable for $\lambda \simeq 0.85$. For very slow running [ $\left.(b-\delta b) \alpha_{\mathrm{c}} \sim 1\right]$, it becomes comparable for $\lambda \simeq 0.7$.

(2) For $\lambda$ between 0 and the above values, $\Sigma(0)$ and $F$ remain essentially unchanged and of order $A_{\text {c }}$. The condensate, however, increases substantially throughout each range. The increase from the $\lambda=0$ value is at first modest, but it is at least three orders of magnitude near the end of each range. In the intermediate running case, the condensate is $\mathrm{O}\left(\Lambda_{c}^{3}\right)$ in the absence of the four-fermion interaction, corresponding to a quark or lepton mass on the order of one $\mathrm{MeV}$. The condensate then increases by nearly an order of magnitude by the time $\lambda$ reaches 0.7 and by more than another two orders of magnitude in the range between 0.7 and 0.85 . At the end of the range, a quark or lepton mass exceeding $1 \mathrm{GeV}$ is generated. In the case of very slow running, the additional effect of the four-fermion interaction can produce a dramatically enhanced condensate within the above range. Near the end, quark and lepton masses in the range of 5$10 \mathrm{GeV}$ can be generated.

(3) In each of the above cases, $\Sigma(p)$ remains small compared to $A$, and $\langle\bar{\psi} \psi\rangle / A^{3} \ll 1$. This suggests that with $\lambda$ restricted to the above ranges, higher dimension operators will lead to even smaller contributions to $\Sigma(p)$ and that the low energy expansion will remain valid ${ }^{\#}$.

(4) For any rate of running, as $\lambda$ is increased beyond the ranges described above, some dramatic effects set in. The changes are very rapid and by the time $\lambda$ exceeds unity, the theory becomes unphysical for technicolor purposes. Consider the intermediate running case $(b-\delta b) \alpha_{c} \sim 0.4$. As $\lambda$ increases from 0.85 to $0.92, F$ grows from being somewhat less than $A_{\mathrm{c}}$ to being several times larger. Since $F$ is the fixed weak scale of the theory, this means that the technicolor confinement scale drops below the weak scale.

\#9 This will presumably require only that the dimensionless coupling strength of the higher dimension operators, normalized to $A$, is no larger than $\lambda$. 
Table 1

The results of a numerical investigation of chiral symmetry breaking, in an SU(4) theory with $N_{f}=8,16,20$ fermion favors, are tabulated for various values of the four-fermion coupling $\lambda \equiv g^{2} / 2 \pi^{2}$. The gauge and fermion contributions to the $\beta$-function are given by $b=44 /$ $6 \pi$ and $\delta b=N_{f} / 3 \pi$, respectively. Since there are $N_{\mathrm{f}} / 2$ weak doublets, $F=250 \mathrm{GeV}\left(N_{\mathrm{f}} / 2\right)^{-1 / 2}$. With $A \approx 2500 \mathrm{TeV}, A / g>A /\left(2 \pi^{2}\right)^{1 / 2} \cong 600$ TeV. The dynamical technifermion mass is $\Sigma(0)$ and the size of $\Sigma(A)$ is a measured of the rate of fall of $\Sigma(p)$. To normalize $F$ relative to $\Sigma(0)$ and $A_{0}$, we have used the relation as described in footnote 8 , which is accurate for the high momentum components. We note that this expression underestimates $f_{\pi}$ in QCD by roughly a factor of two. In the numerical solution of eq. (1), confinement dynamics is approximated crudely by allowing $\alpha(p)$ to plateau at $\alpha\left(A_{\mathrm{c}}\right)=2.5 \alpha_{\mathrm{c}}$ for $p \leqslant A_{\mathrm{c}}$. For $A_{\mathrm{c}}<p<2 \Sigma(0), \alpha(p)$ evolves according to $\beta(\alpha)=-$ $b \alpha^{2}$, and for $p>2 \Sigma(0)$ it evolves according to $\beta(\alpha)=-(b-\delta b) \alpha^{2}$. The output numbers, in particular the dependence on the rate of running and on $\lambda$, are high momentum phenomena and not terribly sensitive to the low momentum approximation for $\alpha(p)$. For values of $\lambda$ small enough so that $A_{\mathrm{c}}$ and $\Sigma(0)$ remain essentially unchanged relative to $A, \alpha(A)$ remains constant. For larger values of $\lambda$, our procedure leads to a small decrease in $\alpha(A)$. This, however, does not play an important role in the results for fermion masses or for the $F / A_{\text {c }}$ hierarchy. In the final column, $\lambda_{\mathrm{Ff}}$ is the two fermion-two technifermion coupling. It is perhaps reasonable to assume $\lambda_{\mathrm{Ft}} \approx \lambda, N_{\mathrm{f}}=8$ case: $F=125 \mathrm{GeV}, b \alpha_{\mathrm{c}}=1.304$ and $\delta b \alpha_{\mathrm{c}}=0.474 ; N_{\mathrm{f}}=16$ case: $F=88 \mathrm{GeV}, b \alpha_{\mathrm{c}}=1.304$ and $\delta b \alpha_{\mathrm{c}}=0.948 ; N_{\mathrm{f}}=20 \mathrm{case}: F=79 \mathrm{GeV}$, $b \alpha_{c}=1.304$ and $\delta b \alpha_{c}=1.185$.

\begin{tabular}{|c|c|c|c|c|c|c|c|c|}
\hline$N_{\mathrm{r}}$ & $\lambda \equiv g^{2} / 2 \pi^{2}$ & $\Sigma(0) / A_{c}$ & $\Sigma(A) / A_{\mathrm{c}}$ & $F / A_{\mathrm{c}}$ & $\langle\bar{y} \mid \psi\rangle / A^{2} A_{\mathrm{c}}$ & $A_{\mathrm{c}}(\mathrm{GeV})$ & \multicolumn{2}{|c|}{$m_{\mathrm{f}} / \lambda_{\mathrm{Ff}}$} \\
\hline \multirow[t]{10}{*}{8} & 0.00 & 0.48 & $8.2 \times 10^{-9}$ & 0.13 & $2.3 \times 10^{-8}$ & 970 & \multicolumn{2}{|c|}{$0.4 \mathrm{MeV}$} \\
\hline & 0.50 & 0.48 & $2.7 \times 10^{-7}$ & 0.13 & $5.0 \times 10^{-8}$ & 970 & \multicolumn{2}{|c|}{$1.0 \mathrm{MeV}$} \\
\hline & 0.70 & 0.48 & $6.9 \times 10^{-7}$ & 0.13 & $9.6 \times 10^{-8}$ & 970 & \multicolumn{2}{|c|}{$1.8 \mathrm{MeV}$} \\
\hline & 0.80 & 0.48 & $1.4 \times 10^{-6}$ & 0.13 & $1.8 \times 10^{-7}$ & 970 & \multicolumn{2}{|c|}{$3.4 \mathrm{MeV}$} \\
\hline & 0.90 & 0.48 & $9.5 \times 10^{-6}$ & 0.13 & $1.0 \times 10^{-6}$ & 970 & 20 & $\mathrm{MeV}$ \\
\hline & 0.91 & 0.48 & $1.9 \times 10^{-5}$ & 0.13 & $2.0 \times 10^{-6}$ & 970 & 39 & $\mathrm{MeV}$ \\
\hline & 0.92 & 0.48 & $3.6 \times 10^{-4}$ & 0.13 & $3.9 \times 10^{-5}$ & 960 & 730 & $\mathrm{MeV}$ \\
\hline & 0.93 & 0.84 & $6.3 \times 10^{-2}$ & 0.26 & $6.6 \times 10^{-3}$ & 480 & 62 & $\mathrm{GeV}$ \\
\hline & 0.94 & 1.6 & 0.30 & 0.77 & $3.2 \times 10^{-2}$ & 160 & 100 & $\mathrm{GeV}$ \\
\hline & 0.95 & 4.1 & 1.5 & 3.2 & 0.16 & 39 & 120 & $\mathrm{GeV}$ \\
\hline \multirow[t]{11}{*}{16} & 0.0 & 0.55 & $3.3 \times 10^{-8}$ & 0.15 & $4.6 \times 10^{-8}$ & 590 & \multicolumn{2}{|c|}{$0.5 \mathrm{MeV}$} \\
\hline & 0.50 & 0.55 & $6.4 \times 10^{-7}$ & 0.15 & $1.1 \times 10^{-7}$ & 590 & \multicolumn{2}{|c|}{$1 \mathrm{MeV}$} \\
\hline & 0.70 & 0.55 & $2.1 \times 10^{-6}$ & 0.15 & $2.8 \times 10^{-7}$ & 590 & \multicolumn{2}{|c|}{$3 \mathrm{MeV}$} \\
\hline & 0.80 & 0.55 & $8.8 \times 10^{-6}$ & 0.15 & $1.0 \times 10^{-6}$ & 590 & \multicolumn{2}{|c|}{$12 \mathrm{MeV}$} \\
\hline & 0.82 & 0.55 & $1.9 \times 10^{-5}$ & 0.15 & $2.2 \times 10^{-6}$ & 590 & \multicolumn{2}{|c|}{$25 \mathrm{MeV}$} \\
\hline & 0.84 & 0.57 & $1.0 \times 10^{-3}$ & 0.15 & $1.2 \times 10^{-4}$ & 570 & \multicolumn{2}{|c|}{$1.3 \mathrm{GeV}$} \\
\hline & 0.86 & 0.78 & $1.9 \times 10^{-2}$ & 0.23 & $2.1 \times 10^{-3}$ & 390 & \multicolumn{2}{|c|}{$17 \mathrm{GeV}$} \\
\hline & 0.88 & 1.1 & $8.2 \times 10^{-2}$ & 0.42 & $8.9 \times 10^{-3}$ & 210 & \multicolumn{2}{|c|}{$37 \mathrm{GeV}$} \\
\hline & 0.90 & 1.9 & 0.31 & 1.0 & $3.3 \times 10^{-2}$ & 85 & \multicolumn{2}{|c|}{$55 \mathrm{GeV}$} \\
\hline & 0.91 & 2.8 & 0.62 & 1.8 & $6.6 \times 10^{-2}$ & 49 & \multicolumn{2}{|c|}{$64 \mathrm{GeV}$} \\
\hline & 0.92 & 4.6 & 1.3 & 3.5 & 0.14 & 26 & \multicolumn{2}{|c|}{$72 \mathrm{GeV}$} \\
\hline \multirow[t]{9}{*}{20} & 0.00 & 0.63 & $4.9 \times 10^{-7}$ & 0.17 & $3.4 \times 10^{-7}$ & 470 & 3 & $\mathrm{MeV}$ \\
\hline & 0.50 & 0.63 & $9.6 \times 10^{-6}$ & 0.17 & $1.5 \times 10^{-6}$ & 460 & 14 & $\mathrm{MeV}$ \\
\hline & 0.55 & 0.63 & $1.6 \times 10^{-5}$ & 0.17 & $2.3 \times 10^{-6}$ & 460 & 21 & $\mathrm{MeV}$ \\
\hline & 0.60 & 0.63 & $3.6 \times 10^{-3}$ & 0.17 & $4.9 \times 10^{-6}$ & 460 & 45 & $\mathrm{MeV}$ \\
\hline & 0.65 & 0.65 & $3.1 \times 10^{-4}$ & 0.18 & $3.9 \times 10^{-5}$ & 450 & 350 & $\mathrm{MeV}$ \\
\hline & 0.70 & 0.75 & $3.2 \times 10^{-3}$ & 0.21 & $4.0 \times 10^{-4}$ & 370 & 3 & $\mathrm{GeV}$ \\
\hline & 0.75 & 0.95 & $1.6 \times 10^{-2}$ & 0.30 & $1.9 \times 10^{-3}$ & 270 & 10 & $\mathrm{GeV}$ \\
\hline & 0.80 & 1.3 & $6.8 \times 10^{-2}$ & 0.54 & $7.7 \times 10^{-3}$ & 150 & 22 & $\mathrm{GeV}$ \\
\hline & 0.90 & 8.4 & 2.6 & 7.0 & 0.27 & 11 & 61 & $\mathrm{GeV}$ \\
\hline
\end{tabular}

The resulting light physics would consist of relatively narrow techni-glueballs which could be produced at existing or soon-to-exist accelerators. With $\lambda=0.92$, for example, the technicolor confinement scale drops to roughly $20 \mathrm{GeV}$, compared to $F=90 \mathrm{GeV}$, $\Sigma(0)=120 \mathrm{GeV}$, and the condensate is large enough to produce a quark or lepton mass greater than 50 $\mathrm{GeV}$. In general, a fermion mass large enough to ac- 
commodate the $t$ quark emerges in the range of $\lambda$ values that can lead to physics below the weak scale. While the low energy expansion still appears to remain valid for $\lambda$ in this range, the results are becoming more sensitive to physics at $A$. It will be important to explore this sensitivity in more detail.

A natural question is whether there is an expected range of values of $\lambda$ in technicolor theories. It is clear from table 1 that substantial condensate enhancement, due partly to the four-fermion interaction, occurs for reasonably large range of $\lambda$ especially if there are enough fermions to slow the running of the coupling somewhat. An enhancement large enough to produce a $t$ quark mass and light techniglueballs, on the other hand, emerges only for a narrower range of $\lambda$ values nearer to unity.

In this letter, we offer only a few general remarks on expectations for the size of $\lambda$. If the four-fermion interactions are due to ETC boson exchange, it would not be unreasonable to speculate that $\lambda$ should be of order $\alpha(\Lambda)$, the technicolor coupling at the ETC scale. This would be expected if these interactions become unified at $A$. In the SU (4) examples of table $1, \alpha(\Lambda)$ ranges between 0.2 and 0.35 , somewhat less than the "interesting" range of values of $\lambda$. On the other hand, $\lambda$ contains factors depending on the unified theory and therefore not present in the technicolor coupling $\alpha(\Lambda)$. Because the unified group is larger than the technicolor group, these factors might be expected to be larger than unity. This will be examined in more detail in a future publication.

Whatever the source of the four-fermion interactions, it should be clear from the above analysis that a viable electroweak theory does not seem possible if $\lambda>1$. This could prove to be a stumbling block for theories in which the four-fermion interactions are imagined to arise from a composite structure for the fermions and technifermions.

While the appearance of fermion masses up into the $\mathrm{GeV}$ range is welcome, the problem of producing a realistic quark and lepton mass spectrum remains. In technicolor theories, the mysteries of the large hierarchies in the mass spectrum get shifted into the effective four-fermion interactions among the technifermions and the quarks and leptons. It should be clear from the above analysis, however, that this mapping can be far from linear. In particular, a small breaking of weak isospin or flavor symmetry in the four-technifermion couplings can be considerably magnified in the quark and lepton mass spectrum.

We have studied this magnification effect in more detail in a simple two-coupling example constructed from the SU(4) theory by including $N_{\mathrm{f}}=16$ technifermions divided into two families of eight. With fourtechnifermion couplings $\lambda_{1}$ and $\lambda_{2}$ for the two families, a variety of hierarchies will appear depending on the relative size of $\lambda_{1}$ and $\lambda_{2}$. In particular, for $\lambda_{1} \ll \lambda_{2} \lesssim 1, F_{2}$ and $\Sigma_{2}(0)$ can be on the order of the weak scale while $F_{1}, \Sigma_{1}(0)$ and the confinement scale can be smaller. Thus, there could be some relatively light technimesons and baryons along with light techni-glueballs. A smaller difference between $\lambda_{1}$ and $\lambda_{2}$ will keep all the above scales on the order of the weak scale and yet still lead to a large ratio between the condensates of family one and family two. If each technifamily then couples to an ordinary quark-lepton family, a large family hierarchy for the quark and lepton masses will emerge. An important point is that this hierarchy can develop without a hierarchy of ETC scales, but instead from modest coupling differences at a single ETC scale.

The large amount of weak isospin breaking in the quark and lepton mass spectrum poses a special problem for technicolor theories. Not only must a sufficiently large $\mathrm{t}-\mathrm{b}$ quark mass splitting be generated, but this must be done in a way that does not overly infect the experimentally measured $\rho$ parameters $\# 10$. The fact that $\rho-1 \equiv M_{\mathrm{W}}^{2} / M_{\mathrm{Z}}^{2} \cos ^{2} \theta_{\mathrm{W}}-1<$ $10^{-2} \# 11$, means that very little weak isospin violation can be allowed in $\rho$. We have begun an analysis of this problem by including an isospin violating fourfermion coupling $\lambda_{3}$ along with the symmetric coupling $\lambda$. To lowest order in electroweak interactions, $\rho=F_{ \pm}^{2} / F_{0}^{2}$, where $F_{ \pm}$and $F_{0}$ are the decay constants for the charged and neutral Goldstone bosons. A weak isospin violating deviation of this ratio from unity will be induced by the $\lambda_{3}$ interaction, but unless it becomes quite strong, it will not violate the above experimental constraint. Nevertheless, a somewhat smaller $\lambda_{3}$ can induce a healthy isospin violation in the condensate, leading to realistic up-down fermion mass splittings. Whether the $t-b$ splitting can be ac-

\footnotetext{
\#10 This point has been stressed in a recent paper by Chivukula [13].

\#11 For a recent review see ref. [14].
} 
commodated using the same value of $A$ and without upsetting the $\rho$ parameter is not yet clear.

Finally, it is worth remarking that small amounts of weak isospin breaking will be induced by the electroweak interactions. The magnification effect we have described could then mean that these small increments to the four-fermion couplings could produce large weak isospin splitting in the quark and lepton mass spectrum. QCD effects could be magnified in a similar way, leading to important contributions to quark-lepton mass differences ${ }^{\sharp 12}$. There will be analogous contributions to the masses of possible pseudo-Goldstone bosons. In general, the new interactions at scale $\Lambda$, if they are strong enough, can add to and magnify the contributions of standard model gauge interactions to the mass parameters of the standard model and its extensions.

This work is supported in part by the US Department of Energy under contract number of DE-AC02ERO-3075 and by an O.J.I. Grant of DOE channeled through the contract FG-0284-ER-40153. L.C.R.W. acknowledges a Faculty Research Fellowship from the University of Cincinnati. The hospitality of the Aspen Center for Physics, where this work was begun, is also acknowledged. Conversations with W. Bardeen, S. Chivukula, B. Holdom, L. Krauss, K. Lane, and M. Soldate are greatly appreciated.

\#12 It has also been pointed out by Holdom that in the presence of a slowly running technicolor coupling, QCD contributions to quark-lepton mass differences can be enhanced [15]. He also noted that symmetry breaking effects at the ETC scale could produce condensate hierarchies for technifermions when the gauge coupling runs slowly. Our analysis shows that this can happen for normally running theories as well, when the four fermion coupling is sufficiently strong.

\section{References}

[1] T. Masakawa and H. Nakajima, Prog. Theor. Phys. 52 (1974) 1326; 54 (1976) 860;

R. Fukuda and T. Kugo, Nucl. Phys. B 117 (1976) 250;

K. Higashijima, Phys. Rev. D 29 (1984) 1228;

P. Castorina and S.Y. Pi, Phys. Rev. D 31 (1985) 411;

R. Casalbuoni, S. De Curtis, D. Dominici and R. Gatto, Phys. Lett. B 150 (1985) 295.

[2] T. Appelquist, K. Lane and U. Mahanta, Phys. Rev. Lett. 61 (1988) 1553.

[3] S. Dimopoulos and L. Susskind, Nucl. Phys. B 155 (1979) 237;

E. Eichten and K. Lane, Phys. Lett. B 90 (1980) 125.

[4] R. Kaul, Rev. Mod. Phys. 55 (1983) 449.

[5] T. Appelquist, D. Karabali and L.C.R. Wijewardhana, Phys. Rev. Lett. 57 (1986) 957;

T. Appelquist and L.C.R. Wijewardhana, Phys. Rev. D 35 (1987) 774; D 36 ( 1987$) 568$.

[6] B. Holdom, Phys. Rev. D 24 (1981) 1441.

[7] B. Holdom, Phys. Lett. B 150 (1985) 301.

[8] T. Appelquist, D. Carrier, L.C.R. Wijewardhana and W. Zheng, Phys. Rev. Lett. 60 (1987) 1114.

[9] T. Akiba and Y. Yanagida, Phys. Lett. B 169 (1986) 432; K. Yamawaki, M. Bando and K. Matumoto, Phys. Rev. Lett. 56 ( 1986 ) 1335; Phys. Lett. B 178 (1986) 308.

[10] Y. Nambu and G. Jona-Lasinio, Phys. Rev. 122 (1961) 345.

[11] H. Pagels and S. Stokar, Phys. Rev. D 20 (1979) 2947.

[12] T. Appelquist, M. Soldate, T. Takeuchi and L.C.R. Wijewardhana, YCTP-P19-88, in: Proc. 12th Johns Hopkins Workshop on Current problems in particle theory (Baltimore, MD, June 1988), to be published.

[13] R.S. Chivukula, Phys. Rev. Lett. 61 (1988) 2657.

[14] P. Langacker, invited talk at the XXIV Intern. Conf. on High energy physics (Munich, August, 1988); and the 1988 Meeting of the Division of Particles and fields of the APS (Storrs, CT, August 1988).

[15] B. Holdom, Phys. Rev. Lett. 60 (1988) 1233. 